\title{
The Role of Migration in the Development of Depressive Symptoms among Latino Immigrant Parents in the USA
}

\author{
India J. Ornelas[Acting Assistant Professor] and \\ Department of Health Services University of Washington \\ Krista M. Perreira[Associate Professor] \\ Department of Public Policy and Carolina Population Center University of North Carolina at \\ Chapel Hill
}

\begin{abstract}
Nearly one out of every four children in the US is a child of immigrants. Yet few studies have assessed how factors at various stages of migration contribute to the development of health problems in immigrant populations. Most focus only on post-migration factors influencing health. Using data from the Latino Adolescent Migration, Health, and Adaptation Project, this study assessed the extent to which pre-migration (e.g., major life events, high poverty), migration (e.g., unsafe and stressful migration experiences), post-migration (e.g., discrimination, neighborhood factors, family reunification, linguistic isolation), and social support factors contributed to depressive symptoms among a sample of Latino immigrant parents with children ages 12-18. Results indicated that high poverty levels prior to migration, stressful experiences during migration, as well as racial problems in the neighborhood and racial/ethnic discrimination upon settlement in the US most strongly contribute to the development of depressive symptoms among Latino immigrant parents. Family reunification, social support, and familism reduce the likelihood of depressive symptoms.
\end{abstract}

\section{Keywords}

USA; migration; family; Latino; Hispanic; immigrant; depression; acculturation

\section{Introduction}

Research shows that foreign-born Latinos living in the United States (US) have lower rates of depressive symptoms and other mental health problems than US born Latinos and nonLatino Whites (Alegria, Shrout, Woo, Guarnaccia, Sribney, Vila et al., 2007; Grant, Stinson, Hasin, Dawson, Chou, \& Anderson, 2004; Ortega, Rosenheck, Alegria, \& Desai, 2000). Latino immigrants' rates of mental health disorders also tend to increase with length of residence in the United States (Cook, Alegria, Lin, \& Guo, 2009; Nicklett \& Burgard, 2009). Many have attributed this pattern to the negative impact of post-migration stressors. However, few studies have explored the extent to which factors prior to migration and the migration experience itself influence mental health. Given the continued growth of the

(C) 2011 Elsevier Ltd. All rights reserved.

Corresponding Author: Krista M. Perreira, perreira@email.unc.edu.

Publisher's Disclaimer: This is a PDF file of an unedited manuscript that has been accepted for publication. As a service to our customers we are providing this early version of the manuscript. The manuscript will undergo copyediting, typesetting, and review of the resulting proof before it is published in its final citable form. Please note that during the production process errors may be discovered which could affect the content, and all legal disclaimers that apply to the journal pertain. 
Latino immigrant population, public health researchers and mental health care providers must develop a more comprehensive understanding of the factors that contribute to poor mental health in immigrant families.

In this paper, we examine the role of pre-migration, migration and post-migration experiences in the development of depressive symptoms among immigrant parents. We focus on immigrant parents because of the strong relationship between parental mental health and child mental health (Beeber, Holditch-Davis, Perreira, Schwartz, Lewis, Blanchard et al., 2009). The well-being of immigrant parents is not only important in its own right but has significant consequences for the well-being and development of the children of immigrants.

\section{The Relationship between Migration and Depression}

The migration process encompasses several factors which can threaten individual and family well-being. Following Sluzki's stages of migration framework, we identify three key stages of migration - the pre-migration, migration, and post-migration stages - that provide a template for understanding sources of stress throughout the migration process and the mental health consequences of these stressors (Ko \& Perreira, 2010).

Pre-Migration Experiences and Depression-In the pre-migration stage, parents make a decision to leave their home countries. These decisions typically reflect economic hardships in their home countries, political unrest and persecution, or the desire to reunify with family already living in the US. Although theory suggests that immigrants' experiences in their home countries prior to migration can impact their post-migration mental health, few empirical studies have confirmed these relationships. To date studies have found that low income, low levels of education, and low social status in their home countries can increase immigrants' risks for depressive symptoms and major depressive episodes (Aguilar-Gaxiola \& Gullota, 2008; Nicklett \& Burgard, 2009). In addition, experiencing political violence or other traumatic events can have long-term mental health consequences for immigrants (Fortuna, Porche, \& Alegria, 2008).

Migration Experiences and Depression-The migration stage captures the process of migrating including the hardships experienced during travel. Due to recent changes in immigration policy, including increased immigration enforcement along the US-Mexico border, many immigrants from Mexico enter the US under dangerous conditions and without legal documentation (Massey, Durand, \& Malone, 2002). Furthermore, migrants from Latin America are increasingly settling in new immigrant destinations in the Southern and Midwestern US, increasing the time spent in transit and thus, the potential for facing adversity (Kandel \& Parrado, 2005; Kochkar, Suro, \& Tafoya, 2005). During their travels, they experience traumatic events, such as sexual and physical abuse, robbery, and illness, and are vulnerable to exploitation (DeLuca, McEwen, \& Keim, 2010; Moynihan, Gaboury, \& Onken, 2008; Rasmussen, Rosenfeld, Reeves, \& Keller, 2007; Sladkova, 2007). Consequently, migration can be quite stressful, especially for parents traveling with children or concerned about family members left behind (Cavazos-Rehg, Zayas, \& Spitznagel, 2007; DeLuca et al., 2010). Yet few studies have documented how migration experiences contribute to depressive symptoms among adult Latino immigrants, especially parents (Perreira \& Potochnick, 2010).

Post-Migration Experiences and Depression-The post-migration stage pertains to settlement experiences, the process of navigating life in a new country, changes in family structure, and neighborhood environment. Previous studies have identified various stressors associated with immigrant adaptation which contribute to depressive symptoms, including 
racial/ethnic discrimination, aspects of the neighborhood environment, and language barriers. Other factors such as family reunification can potentially reduce the risk of depressive symptoms.

Once they arrive in the US, immigrants' skin color, culture, and language use can place them at risk for experiencing racial/ethnic discrimination, especially in new immigrant destinations in the Midwest and Southeast where they face increased exposure to antiimmigrant sentiment (Marrow, 2009). Latino immigrants report higher rates of discrimination than US-born Latinos (Finch, Kolody, \& Vega, 2000). Several studies have linked this discrimination to poor mental health (Cook et al., 2009; Finch et al., 2000; Gee, Ryan, Laflamme, \& Holt, 2006). However, few have assessed its impact among Latino immigrant parents or relative to other migration factors.

Among other post-migration factors expected to influence the mental health of immigrants, we consider the composition and safety of parents' neighborhoods. Although some studies have found that Latinos living near other Latinos have better mental health because coethnic neighborhoods provide them with social support and access to resources through social networks, co-ethnically dense neighborhoods can also have disadvantages which increase the risk for poor mental health outcomes including depression (Acevedo-Garcia \& Lochner, 2003; Alegria et al., 2007; Brown, et al., 2009; Lee, 2009; Ostir, Eschbach, Markides, \& Goodwin, 2003). In dense co-ethnic immigrant communities, some immigrants -- particularly women -- can experience increased stress due to expectations of reciprocity and obligations to provide instrumental social support to more recent immigrants (Kao, 2004; Menjivar, 2000; Parrado, Flippen, \& McQuiston, 2005). Others may feel racially segregated in these neighborhoods, have reduced access to economic opportunities, and have increased exposure to stressors such as crime and poverty (Feldmeyer, 2009; Iceland \& Scopilliti, 2008). Because many are learning how to navigate life in the US and interacting with other racial/ethnic groups for the first time, foreign-born Latinos may be more sensitive to segregation and racial/ethnic conflicts than their US counterparts (Perreira, Chapman, \& Stein, 2006). Yet few researchers have assessed these relationships among Latino immigrants.

Studies have shown that language barriers are also a common stressor among Latino immigrants (Kim-Goodwin \& Bechtel, 2004). While many immigrants living in established co-ethnic Latino communities can function effectively using Spanish only, over time language barriers can limit their economic and social mobility (Tienda \& Mitchell, 2006). For immigrants living in new immigrant and emerging Latino communities with few Spanish-speaking resources, the stress associated with language barriers can be compounded. Consequently, language barriers and English competency pressures can lead to higher rates of depressive symptoms, especially among immigrants who have been in the US ten years or less (Ding \& Hargraves, 2009; Torres, 2010). Language barriers can also impact immigrants' access to and utilization of health services, which can result in less treatment and care for those struggling with mental health problems (Cabassa, Zayas, \& Hansen, 2006; Sentell, Shumway, \& Snowden, 2007).

Lastly, the opportunity to reunite with family members is a post-migration factor that can positively influence the mental health of Latino immigrants. Many parents are forced to leave children or other family members behind in their home countries when they migrate. Separation from children and spouses is known to contribute to depression among Mexican immigrant mothers (Ornelas, Perreira, Beeber, \& Maxwell, 2009; Suarez-Orozco, Todorova, $\&$ Louie, 2002). Therefore, parents who are able to reunite with family members after migration can have a lower risk of developing depressive symptoms. 


\section{Social Support Factors and Depression}

Despite the prevalence of several stressors before, during, and after migration, social support from friends and family can buffer immigrants from the development of depressive symptoms at each of these stages by reducing the frequency, intensity or duration of stressors (Heaney \& Israel, 2008). Studies have shown that Latino immigrants with higher levels of social support have lower levels of stress and fewer depressive symptoms and that a lack of social support is associated with increased depressive symptoms (Brown et al., 2009; Falcon, Todorova, \& Tucker, 2009; Hovey, 2000a, b).

Familism, a strong connection and loyalty to family members, is especially important among Latinos and may serve as another form of social support (Kuperminc, Wilkins, Roche, \& Alvarez-Jimenez, 2009). High levels of family social support reduce their risk of psychological distress (Rivera, 2007; Vega, Kolody, Valle, \& Weir, 1991). Conversely, low levels of familism and high levels of family conflict and dysfunction are related to poorer mental health (Alegria et al., 2007; Cook et al., 2009 Hovey, 1999; Hovey \& Magana, 2000; Rivera, et al., 2008).

Therefore, based on previous research related to Latino immigrant mental health, the primary aim of our study was to assess whether pre-migration, migration, and postmigration factors contribute to the development of depressive symptoms among Latino immigrant parents. We also examined whether social support, and familism protect against the development of depressive symptoms in the face of migration-related stressors.

\section{Methods}

\section{Study design and sample}

We used data from the Latino Adolescent Migration, Health, and Adaptation Project (LAMHA), a population-based study of mental health, migration and acculturation among 281 first-generation Latino youth and their parents in North Carolina. Between 1990 and 2000, North Carolina experienced tremendous growth in its Latino immigrant population and represents the current demographic trend of immigrants settling in new destination communities. Data for the LAMHA study were collected from $2004-2006$ using a stratified-cluster sampling design to survey youth and their primary caregivers. Data collection was stratified by rural-urban counties and clustered by middle or high school. The present study uses data from 281 caregivers, which represents Latino immigrant parents of middle and high school students in North Carolina. The caregivers interviewed were mostly biological mothers (76\%); biological fathers (14\%) or other relatives (10\%) responsible for the adolescent's guardianship when no mother was present in the household. Due to low literacy levels in the population, the parent survey was interviewer-administered in the parents' preferred language (English or Spanish). Most of the sample was from Mexico (78\%) and the majority (96\%) completed the survey in Spanish. Aside from Mexico, our participants immigrated from El Salvador (4\%), Honduras (6\%), and other Central American (3\%), South American (6\%), or Caribbean countries (3\%). Further details on the survey and sampling design have been fully described elsewhere (Perreira, Chapman, Potochnick, \& Smith, 2008). The study was approved by the Institutional Review Board of the University of North Carolina at Chapel Hill.

\section{Measures}

Depressive Symptoms-We assessed depressive symptoms with two measures validated and extensively used among Spanish-speaking adults - the PHQ-9 and the CES-D. The Patient Health Questionnaire-9, a modified version of the Prime-MD, is a 10-item measure developed to screen for the frequency of depressive symptoms in clinic settings 
(Kroenke \& Spitzer, 2002). PHQ-9 scores can provide a tentative diagnosis of mild to severe depression. The Center for Epidemiological Studies Depression Scale (CES-D) is a 20-item measure designed to assess depressive symptoms in epidemiological studies rather than clinic settings (Radloff, 1977).

For the PHQ-9, respondents were asked to think about the past 14 days and reported how often they have been bothered by a list of items during that time period. The list of items is based on the DSM-IV (American Psychiatric Association, 1994) criteria for major depressive disorder, for example, "Feeling down, depressed or hopeless." Response options for the first nine questions ranged from "Not at all" (0) to "Nearly every day" (3) and items were summed to produce a score ranging from 0 to 27 with higher scores indicating more frequent depressive symptoms ( $\alpha=.83$, all alphas reported were calculated for the study sample). The tenth question is included to assess severity of symptoms and asks individuals to indicate how difficult these problems have made it for them to do work, take care of things at home, or get along with other people. Individuals must respond that these problems have been at least somewhat difficult for their symptoms to be considered clinically significant and in need of treatment. Because few study participants met the criteria for major depression (i.e. a score of 15+), we created an indicator variable for those with a score of five. This lower score indicates at least mild depressive symptomatology warranting further evaluation by a physician (Kroenke \& Spitzer, 2002).

For the CES-D, respondents are asked to indicate how often a list of statements has been true over the past seven days. For example, "I felt depressed," with responses range from "<1 day/wk" (1) to "5-7 days/wk" (4). Items are summed from 0 to 60 with higher scores indicating more frequent depressive symptoms $(\alpha=.90)$. An indicator variable was created for those with scores of 16 or higher which indicates clinically significant psychological distress and is correlated with a diagnosis of depression (Radloff 1977).

In the data collection, questions from the PHQ-9 preceded CES-D questions. Thus, respondents were first instructed to think about their feelings during the past 2 weeks and then consider only the past week. By evaluating responses to both the PHQ-9 and the CES$\mathrm{D}$, we were able to gauge the robustness of our results to different types of depressive symptoms as well as their severity. In our sample, the PHQ-9 allows for the identification of mild depression based on DSM-IV criteria and focuses exclusively on negative affect (e.g., feeling down, depressed, or hopeless) and somatic complaints (e.g., poor appetite or overeating). The CES-D contains additional items on positive affect which are reverse coded (e.g., I was happy) and items on interpersonal relationships (e.g., I felt that people disliked me) which can signal dysphoria or anhedonia as well as depression. The correlation between PHQ-9 scores and the CES-D scores in our sample was .76, a level of correlation similar to correlations in other studies using both instruments (Milette et al., 2010).

Pre-Migration Factors-We included two pre-migration factors in our analysis. Parents were asked whether they experienced any of the following major life events prior to their migration: respondent or spouse lost a job or business, natural disaster, family member or friend was arrested or killed, or another traumatic event specified by the respondent. Parents were also asked whether escaping violence or persecution was their primary reason for migrating. We then created an indicator variable for parents who had experienced any major life event, trauma, violence, or persecution prior to migration. Following guidelines for measuring poverty in Mexico (CONEVAL, 2010), we also created an indicator variable for high poverty ( $0=$ no, $1=$ yes) which included those who had dirt floors or lacked indoor plumbing prior to migrating to the US. 
Migration Factors-We assessed four aspects of the migration process. First, parents were asked if they were ever concerned for their safety during their travels to the US, and an indicator variable was created $(0=$ no, $1=$ yes $)$. Second, parents were asked how stressful their move to the US was -- "not at all," "somewhat," or "very." An indicator variable was created for those who reported that their migration was very stressful. Third, we asked parents whether they experienced any of the following traumatic events during their migration: robbery, physical attack, accidental injury, illness, or another traumatic event specified by the respondent. We then created an indicator variable for parents who had experienced any traumatic event during migration. Fourth, respondents were asked what kind of documentation or visa they had when they first entered the US. Those who entered without legal documents were coded as, "entered without documentation."

Post-migration Factors-We measured four potential post-migration stressors. Racial/ ethnic discrimination was assessed with one item asking respondents if they had ever been discriminated against in the US because of their race or ethnicity ( $0=$ no, $1=y e s)$. Participants' were also asked which racial/ethnic groups discriminate against them most often and the types of discrimination that they experienced. Neighborhood racial conflict was assessed with one item asking whether racial or cultural groups not getting along with each other was a problem in their neighborhood $(0=$ no, $1=y e s)$. Compatriots live in my neighborhood was assessed with one item asking respondents whether many people from their country lived in their neighborhood $(0=$ no, $1=y e s)$. Parents were coded as having an unsafe neighborhood if they responded that any of the following were a problem in their neighborhood: little respect for rules, laws, and authority; assaults and muggings; delinquent gangs or drug gangs; or, drug use or drug dealing out in the open. We also created an indicator variable for those who responded that they spoke and read only Spanish. Finally, respondents were asked whether they had migrated together with the children currently living in their household or had been separated from them after migrating to the US. An indicator variable for family reunification was created for respondents who initially had been separated from their children but had since been reunited ( $0=$ no, $1=y e s)$. Respondents who had been separated from their children were asked how many months they had been separated.

Social Support Factors-Social support was assessed with the 12-item Interpersonal Support Evaluation List (ISEL-12), a measure commonly utilized in studies with Spanishspeaking populations. It asks respondents about their perceptions of the availability of various types of support, such as practical help, advice and companionship; for example, "When I need suggestions about how to deal with a personal problem, I know someone I can turn to" (Cohen \& Hoberman, 1983; Cohen \& Wills, 1985). Response options range from definitely false (1) to definitely true (4). All items were summed to create a global measure of social support with scores ranging from 12 to 48 and higher scores indicating greater social support $(\alpha=.77)$. Familism was assessed with a seven item scale including questions related to family cohesion and loyalty; such as, "We share similar values and beliefs as a family" (Gil \& Vega, 1996). Response options were on a five point Likert scale with scores ranging from 7 to 35 and higher scores indicating a higher level of familism $(\alpha=.90)$.

Demographic Variables-We assessed several demographic variables including age, gender, marital status, education, employment status, length of residence in the US and acculturation. Age was measured in years and an indicator variable was created for those married or living as married. Education was categorized as, "Less than $8^{\text {th }}$ grade," " 8 th grade to high school," "High school graduate," and "College graduate." An indicator variable was created for those that were currently employed full or part-time. Length of residence in the US was measured in years. In our study, acculturation was viewed as a two-dimensional 
construct capturing an individual's sense of attachment and belonging with either AngloAmerican, Latino/Hispanic cultures or both. We measured acculturation using the ten item Psychological Acculturation Scale (PAS) (Tropp, Erkut, Coll, Alarocon, \& Vazquez Garcia, 1999). Response options ranged from "Latinos only" (1) to "Anglos only" (5) and were averaged for a summary score $(\alpha=.88)$.

\section{Data Analysis}

With few exceptions, we hypothesized that each pre-migration, migration, and postmigration related stressor would be positively associated with depressive symptoms. Given the mixed evidence regarding co-ethnic neighborhoods and mental health, we did not make hypotheses about the direction of its relationship with depressive symptoms. Family reunification, social support, and familism were hypothesized to protect against depressive symptoms in the presence of migration-related stressors.

To test these hypotheses, we first examined the characteristics of the study sample using univariate descriptive statistics. We estimated crude odds ratios and $95 \%$ confidence intervals for the PHQ-9 and CES-D for all the independent variables and socio-demographic covariates using logistic regression. Then we constructed four sets of age and gender adjusted models for each outcome variable. To maintain a consistent sample size across models, we dropped 35 cases with missing data on at least one study variable. There were no significant differences between those with and without missing data for the demographic or other independent variables. Age and gender were selected as covariates based on previous research showing differences in depressive symptoms on these factors. No other demographic covariates significantly influenced the risk of depression in our sample. Therefore, they were not included in our final results. The first set (Model 1) included only the pre-migration factors, the second (Model 2) included only the migration factors, the third included only post-migration factors (Model 3 ) and the fourth included only the social support factors (Model 4). Finally, we estimated fully adjusted models for each outcome which included all the significant independent variables from partially adjusted models 1, 2, 3 , and 4 . All estimations adjust for the stratification and clustering of the data.

\section{Results}

Table 1 shows the distribution of key variables. The sample was mostly female (84\%) with a mean age of 40. Most were married, employed and had less than a high school education. The average length of residence in the US was 8 years with mean acculturation score of 1.8. Thus, our sample continued to most strongly affiliate with Latino values, beliefs, and culture even after an average of 8 years in the US. Few (25\%) of our respondents had lived in the US fewer than 5 years and only $27 \%$ had lived in the US over 10 years. Over $26 \%$ reported having minimal depressive symptoms based on the PHQ-9 and 14\% reported substantial depressive symptoms based on the CES-D.

Several participants experienced major life events prior to migration (30\%), but few had lived in high poverty (7\%). The majority of the sample reported experiencing stressors during their migration to the United States. Almost all feared for their safety during their migration, half reported that their migration was very stressful, and $13 \%$ experienced a traumatic event, such as being robbed or physically attacked, during their migration. Over two-thirds of the sample entered without documentation. Migration stressors were somewhat associated with each other. For example, those who characterized the move to the US as very stressful were more likely to have feared for their safety during migration $(\mathrm{OR}=11.14$, $\mathrm{p}<.01)$, experienced a traumatic event during migration $(\mathrm{OR}=3.64, \mathrm{p}<.05)$, or entered without documentation $(\mathrm{OR}=3.12, \mathrm{p}<.05)$. 
In terms of stressors experienced after their migration, almost a third of the sample reported experiencing racial/ethnic discrimination and $17 \%$ reported racial problems in their neighborhood. Of those that experienced discrimination, the majority reported that either Whites (44\%) or African-Americans (24\%) discriminated against them most often. But intra-ethnic discrimination among Latinos of different countries of origin also occurred (14\%). In a typical year, $20 \%$ of the sample reported being treated as less competent or being subject to rude remarks at work and $25 \%$ reported being unwelcome or provided with poor service in public.

Most lived in neighborhoods with other people from their same country and spoke and read only Spanish. Among those living in these co-ethnic neighborhoods, $75 \%$ indicated that their neighbors were relatives from their home country. Among all respondents, $41 \%$ had migrated to the US while leaving their children behind and had subsequently re-united with them. On average, respondents separated from their children when they migrated to the US were separated for 3 years; $75 \%$ were separated for at least 12 months. Post-migration stressors, as with migration stressors, were somewhat associated with one another. For example, those who had experienced racial/ethnic discrimination were more likely to have also reported racial/ethnic problems in their neighborhoods $(\mathrm{OR}=2.99, \mathrm{p}<.05)$. Average scores for social support and familism were high and were also modestly correlated with one another $(\mathrm{r}=.15, \mathrm{p}<.05)$.

Because the sample was relatively homogenous (i.e. mostly recent immigrants from Mexico), none of the socio-demographic characteristics was significantly associated with increased depressive symptoms as measured by either the PHQ-9 or the CES-D. All of premigration and migration-related factors were associated with either the PHQ-9 or the CES-D in the crude analysis (Table 1). Experiencing major life events and high poverty were both associated with the CES-D. Those who felt their move to the US was stressful, feared for their safety during migration, experienced a traumatic event during migration, or entered the US without documentation were more likely to report depressive symptoms. Though their magnitude and significance differed somewhat between outcome measures, the results were consistent.

In the analysis of post-migration factors, each factor considered also achieved significance. However, the magnitude and level of significance varied depending on the indicator of depression used. Racial/ethnic discrimination was associated with the PHQ-9 and having racial problems in one's neighborhood was associated with higher scores on the CES-D. Living near compatriots was associated with increased depressive symptoms according to both the PHQ-9 and CES-D. Living in an unsafe neighborhood and speaking and reading only Spanish was associated with increased depressive symptoms as measured by the CESD. Family reunification was associated with fewer depressive symptoms as measured by the PHQ-9. We also calculated crude odds ratios for the social support variables. Increased social support was inversely associated with the occurrence of depressive symptoms on the PHQ-9 and familism was inversely associated with the CES-D.

We then estimated four sets of adjusted models for both the PHQ-9 and the CES-D. The first set (Table 2, Models 1a-b) included the pre-migration independent variables. In these models, experiencing a major life event prior to migration was associated with increased depressive symptoms on both the PHQ-9 and CES-D. High poverty prior to migration was also associated with depressive symptoms as measured by the CES-D. The second set (Table 2 , Models 2a-b) included all migration-related variables. In the model utilizing the PHQ-9, both a stressful move to the US and experiencing a traumatic event during migration were significantly associated with depressive symptoms. In the model utilizing the CES-D, racial problems in the neighborhood and entering without documentation were significantly 
associated with increased odds of depressive symptoms. The third set of models (Table 2, Models 3a-b) included all the post-migration factors. In the model based on the PHQ-9, discrimination was associated with increased odds of depressive symptoms and family reunification was associated with decreased odds of depressive symptoms. In the model based on the CES-D, experiencing racial problems in the neighborhood and speaking and reading only Spanish was associated with increased depressive symptoms. The fourth set of models included both social support variables (Table 2, Models 4a-b). Social support was inversely associated with depressive symptoms as measured by the PHQ-9 and familism was inversely associated with depressive symptoms as measured by the CES-D.

The full models were estimated to compare the relative impact of pre-migration, migration, post-migration, and social support factors (Table 3). In the PHQ-9 model, a stressful move to the US, experiencing a traumatic event during migration, and discrimination were associated with significant increased risk of depressive symptoms, and social support continued to have a modest protective association. In the CES-D model, high pre-migration poverty and neighborhood racial conflicts were the only stressors associated with increased depressive symptoms, and familism continued to have a modest protective association.

\section{Discussion}

This study has several strengths and makes a substantial contribution to the literature on the mental health of Latino immigrant parents. First, our analysis focuses exclusively on immigrants. This allows us to comprehensively examine the influence of stressors at various stages of migration on the well-being of immigrants. Second, our analysis utilizes two alternative measures of depressive symptoms - the PHQ-9, a measure based on the 9criterion symptoms of a major depressive disorder, and the CES-D, a longer measure widely used in epidemiological studies which captures dysphoria as well as more significant depressive symptoms. Third, our analysis focuses on an immigrant population for whom little data currently exist - Latino immigrants living in new receiving communities. Though our analysis focuses on immigrants in only one state with new receiving communities, the results can be informative to researchers and health practitioners in other states with emerging immigrant and Latino populations.

\section{The Lasting Impact of Pre-Migration and Migration Factors on Depressive Symptoms}

Our study is among the first to examine the impact of pre-migration stressors on depressive symptoms among Latino immigrants. We found that both experiencing major life events and living in high poverty before migration were associated with increased depressive symptoms in crude and adjusted models. In models estimating the effects of migration factors only, having a stressful move, experiencing traumatic events and entering without documentation were all strongly associated with increased depressive symptoms. In our model utilizing the PHQ-9, the over five-fold increase in depressive symptoms associated with a very stressful move to the US was even larger in magnitude than the association between discrimination (a post-migration factor) and depressive symptoms. Similarly, in the model for the CES-D, living in high poverty prior to migration was more strongly associated with depressive symptoms than having racial problems in the neighborhood (a post-migration factor).

Given that our sample has lived in the US for an average of 8 years, these results indicate that the influence of stressors experienced before and during migration persists long after Latino immigrants' initial settlement. A traumatic event such as the loss of a family member or a physical or sexual assault can have lasting physical and emotional consequences that need to be managed over time. Likewise, parents who migrate to improve their economic situation may struggle with financial hardship even after arriving in the US, especially if they are working to send home remittances or pay off debts incurred during migration 
(Ornelas et al., 2009). Future research should assess the prevalence and co-occurrence of post-traumatic stress symptoms and depressive symptoms among Latino immigrants.

\section{Post-Migration Racial Relations, Family Reunification, and Depressive Symptoms}

We also found that several post-migration factors were associated with increased depressive symptoms among Latino immigrant parents. Experiencing racial/ethnic discrimination and having racial problems in one's neighborhood were significantly correlated with one another $(\mathrm{r}=.16)$ and strongly associated with increased depressive symptoms in both crude and fully adjusted models. These results are consistent with previous research on the harmful effects of discrimination on mental health (Cook et al., 2009; Finch et al., 2000).

While we do not know the precise nature of racial problems reported by respondents, some may be discriminatory in nature. Recent studies have shown that Latinos living in new destinations such as North Carolina are highly segregated and local residents of small towns with substantial influxes of Latinos are often intolerant of newcomers (Fennelly, 2008). Both the majority White and minority Black populations sometimes view them as an economic or cultural threat (Marrow, 2009). Moreover, their relatively low numbers in new destinations increase the likelihood of cross-race interactions at work and near home. As a result, living near co-ethnic compatriots did not protect immigrants from the harmful effects of racial/ethnic discrimination or racial tensions in their neighborhoods. In fact, living in a neighborhood with others from the same country was significantly associated with depressive symptoms in the crude models.

Together these findings suggest that certain aspects of living in ethnically dense neighborhoods may be detrimental to mental health. Previous research has shown a negative relationship between residential segregation and depressive symptoms among Latino immigrants (Alegria et al., 2007; Lee, 2009). Research also indicates that the presence of extended family members in one's neighborhood can increase demands for instrumental support from immigrant women and reduce their power in family relationships (Parrado et al., 2005). Therefore, neighborhood social ties may not necessarily provide the type of social support that immigrants need to face the challenges of migration. As discussed below, family support and more instrumental social support that extend beyond neighborhood social ties are the essential aspects of support needed to buffer immigrants from the stresses of migration.

Our findings contrast with previous research showing that Latinos living in co-ethnically concentrated areas had lower levels of depressive symptoms (Brown et al., 2009; Mair, Diez Roux, Shen, Shea, Seeman, Echeverria et al., 2009; Ostir et al., 2003). These discrepant findings may reflect methodological differences. First, these studies did not specifically focus on immigrants but on the broader population of both foreign-born and US-born Latinos. Second, these studies focused on older Hispanic adults (45 and older) living in mostly urban areas and established Latino communities which have more resources for new immigrants. Third, these studies used objective measures of co-ethnic concentration (i.e. percent Hispanic in census tract) while our study measured parents' perceptions of their neighborhoods.

Although living near co-ethnics did not necessarily protect immigrants from the challenges of migration, reunification with children did help to reduce parents' risk for depression. Family separation can be a source of anxiety and stress for both parents and their children (Ornelas et al., 2009; Suarez-Orozco et al., 2002). In addition, the process of re-unification can create tension and conflict in families. However, for the caregivers in this study, the joy of reunification with their children clearly trumped the potential for tension and conflict. 


\section{Protective Effect of Family and Social Support}

Our results confirm that social support and familism can buffer immigrants from some of the negative effects of migration-related stressors. However, this support must extend beyond the loose social ties reflected by co-ethnic neighborhoods and provide immigrants with both emotional and instrumental assistance. This has been a consistent and strong finding across studies on the mental health of both foreign-born and US-born Latinos (Falcon et al., 2009; Hovey, 2000a, b; Rivera et al., 2008). To the extent that community-based and social service organizations can supplement these resources and provide instrumental support such as transportation and language services to new immigrants, they can help to promote the parents' mental health.

\section{Comparing Results across Measures of Depressive Symptoms}

Results for both the PHQ-9 and CES-D were generally consistent across the models. Although not all relationships were significant, most were similar in direction and magnitude for both measures of depressive symptoms. Thus, the variation observed across measures may have been impacted by the sample size or by differential reporting biases for the two measures among Latinos. However, different stressors may also be correlated with different levels of depression (i.e. mild vs. severe), duration of symptoms (i.e. 1 week vs. 2 weeks), and types of depressive symptoms (i.e. negative affect vs. positive affect). For example, experiencing a stressful or traumatic migration was significantly associated with only the PHQ-9, which we coded to identify mild depressive symptoms. The most common symptoms reported on the PHQ-9 were feeling tired or having little energy and having problems with sleep. In contrast, entering without documentation and high pre-migration poverty were associated with the CES-D, which captures dysphoria as well as depressive symptoms. The most common symptoms on the CES-D included a lack of positive affect such as happiness, hopefulness, or enjoyment within the past week. Additionally, the CES-D included items regarding interpersonal relationships such as the item "I felt that people disliked me." The PHQ-9 does not include items related to positive affect or interpersonal relationships. Although both instruments are well-validated measures of depressive symptoms in Latino population, the fact that each measure of depressive symptoms was uniquely associated with different pre- and post-migration factors suggests that neither measure perfectly identifies the risk of depression among adult Latino immigrants. Thus, future studies should consider measuring a range of depressive symptoms across different time frames to capture the full impact of migration-related stressors.

\section{Conclusion}

Despite the many strengths of our study, some limitations are important to acknowledge. We were unable to assess temporal relationships with the cross-sectional data available. It is possible that experiences with depression shape participants' perceptions of their premigration, migration, and post-migration experience, and thus our findings actually reflect reverse causality. We were also limited by our single item measures of discrimination and neighborhood factors, future studies should include more comprehensive instruments to more accurately evaluate these constructs. In addition, we did not have data on the mental health of immigrants in our sample prior to their migration to the US. Future studies should evaluate the development of depressive symptoms by sampling populations in immigrants' countries of origin. Our sample of caregivers includes mothers, fathers, and other relatives serving as the primary guardians of adolescent immigrants. Though we found no difference in depressive symptoms by gender or gender differences in the effects of pre-migration, migration, and post-migration factors, relatively few primary caregivers were male. Future research could benefit from the development of samples of male caregivers in immigrant families and the evaluation of gender differences in the effect of migration experiences on mental health. Also, because our sample was comprised mostly of Spanish-speaking 
Mexican immigrants our findings may not be generalizable to other Latino populations. In sum, stressful experiences during migration appear to have long lasting effects on the mental health of Latino immigrants. Furthermore, the experience of becoming a racial/ethnic minority, including exposure to racial/ethnic discrimination and racial conflict with other groups, also damages their mental health during their early settlement. These results suggest that future research should expand data collection efforts to consider factors at multiple stages of migration as potential determinants of immigrants' mental health.

Given the impact of migration-related stressors on mental health, early intervention could help prevent the development of depressive symptoms. Moreover, early intervention with immigrant parents can help to reduce the detrimental impact of depressive symptoms on child development. To help promote the mental well-being of immigrant parents, policy makers and community health providers can work to ensure that mental health coverage is available at Federally-Qualified Health Centers and community-health clinics where many immigrants receive their care. In addition, general practitioners can be encouraged to ask immigrants how stressful their move to the U.S has been and how they are adjusting, and should routinely screen for depression using short, effective diagnostic tools such as the PHQ-9. Finally, community health providers and other organizations serving immigrant communities can take steps to help new immigrants develop strategies to cope with discrimination and racial conflict and find strength in their cultural heritages, families, and broader social networks.

\section{Acknowledgments}

The LAMHA Project was funded by a grant from the William T. Grant Foundation and directed by Krista M. Perreira and Mimi V. Chapman. Persons interested in obtaining LAMHA restricted use data should see http://www.cpc.unc.edu/projects/lamha for further information. The authors would also like to express their appreciation to Paula Gildner for her management of the data collection process; Stephanie Potochnick for her assistance with data cleaning; and all the schools, immigrant families, and adolescents who participated in our research project.

\section{References}

Acevedo-Garcia, D.; Lochner, K. Residential segregation and health. In: Kawachi, I.; Berkman, L., editors. Neighborhoods and health. Oxford University Press; New York: 2003.

Aguilar-Gaxiola, S.; Gullota, T., editors. Depression in Latinos: Assessment, treatment and prevention. Springer; New York: 2008.

Alegria M, Shrout PE, Woo M, Guarnaccia P, Sribney W, Vila D, et al. Understanding differences in past year psychiatric disorders for Latinos living in the US. Social Science \& Medicine. 2007; 65(2):214-230. [PubMed: 17499899]

American Psychiatric Association. Diagnostic Statistical Manual of Mental Disorders. 4th Edition. Author; Washington, DC: 1994.

Beeber L, Holditch-Davis D, Perreira K, Schwartz T, Lewis V, Blanchard H, et al. Short-term in-home intervention reduces depressive symptoms in early head start Latina mothers of infants and toddlers. Research in Nursing \& Health. 2009; 33:60-76. [PubMed: 20043296]

Brown SC, Mason CA, Spokane AR, Cruza-Guet MC, Lopez B, Szapocznik J. The relationship of neighborhood climate to perceived social support and mental health in older Hispanic immigrants in Miami, Florida. Journal of Aging and Health. 2009; 21(3):431-459. [PubMed: 19318605]

Cabassa LJ, Zayas LH, Hansen MC. Latino adults' access to mental health care: a review of epidemiological studies. Administration and Policy in Mental Health. 2006; 33(3):316-330. [PubMed: 16598658]

Cavazos-Rehg PA, Zayas LH, Spitznagel EL. Legal status, emotional well-being and subjective health status of Latino immigrants. Journal of the National Medical Association. 2007; 99(10):1126-1131. [PubMed: 17987916] 
Cohen S, Hoberman H. Positive events and social supports of buffers of life change stress. Journal of Applied Social Psychology. 1983; 13:99-125.

Cohen S, Wills T. Stress, social support, and the buffering hypothesis. Psychological Bulletin. 1985; 98:310-357. [PubMed: 3901065]

Consejo Nacional de Evaluación de la Política de Desarrollo Social (CONEVAL). Metodología para la medición multidimensional de la pobreza en México. 2010. www.coneval.gob.mx/coneval2/htmls/medicion_pobreza/HomeMedicionPobreza.jsp

Cook B, Alegria M, Lin JY, Guo J. Pathways and correlates connecting Latinos' mental health with exposure to the United States. American Journal of Public Health. 2009; 99(12):2247-2254. [PubMed: 19834004]

DeLuca LA, McEwen MM, Keim SM. United States-Mexico border crossing: experiences and risk perceptions of undocumented male immigrants. Journal of Immigrant and Minority Health. 2010; 12(1):113-123. [PubMed: 18850270]

Ding H, Hargraves L. Stress-associated poor health among adult immigrants with a language barrier in the United States. Journal of Immigrant and Minority Health. 2009; 11(6):446-452. [PubMed: 18941893]

Falcon LM, Todorova I, Tucker K. Social support, life events, and psychological distress among the Puerto Rican population in the Boston area of the United States. Aging \&Mental Health. 2009; 13(6):863-873. [PubMed: 19888706]

Feldmeyer B. Immigration and violence: The offsetting effects of immigrant concentration on Latino violence. Social Science Research. 2009; 38(3):717-731. [PubMed: 19856706]

Fennelly, K. Prejudice toward immigrants in the Midwest. In: Massey, D., editor. New faces in new places. Russell Sage Foundation; New York: 2008.

Finch B, Kolody B, Vega W. Perceived discrimination and depression among Mexican-origin adults in California. Journal of Health and Social Behavior. 2000; 41(3):295-313. [PubMed: 11011506]

Fortuna L, Porche MV, Alegria M. Political violence, psychosocial trauma, and the context of mental health services use among immigrant Latinos in the United States. Ethnicity and Health. 2008; 13(5):435-463. [PubMed: 18850369]

Gee GC, Ryan A, Laflamme J, Holt J. Self-reported discrimination and mental health among African descendants, Mexican Americans, and other Latinos in the New Hampshire REACH 2010 Initiative: The added dimension of immigration. American Journal of Public Health. 2006; 96(10): 1821-1828. [PubMed: 17008579]

Gil A, Vega W. Two different worlds: Acculturation stress and adaptation among Cuban and Nicaraguan families. Journal of Social and Personal Relationships. 1996; 13:435-456.

Grant B, Stinson F, Hasin D, Dawson D, Chou S, Anderson K. Immigration and lifetime prevalence of DSM-IV psychiatric disorders among Mexican Americans and Non-Hispanic Whites in the United States. Archives of General Psychiatry. 2004; 61:1226-1233. [PubMed: 15583114]

Heaney, C.; Israel, B. Social networks and social support. In: Glanz, K.; Rimer, BK.; Viswanath, K., editors. Health behavior and health education: Theory, research and practice. Jossey-Bass; San Francisco, CA: 2008.

Hovey JD. Psychosocial predictors of acculturative stress in Central American immigrants. Journal of Immigrant Health. 1999; 1(4):187-194. [PubMed: 16228722]

Hovey JD. Psychosocial predictors of acculturative stress in Mexican immigrants. Journal of Psychology. 2000a; 134(5):490-502. [PubMed: 11034130]

Hovey JD. Psychosocial predictors of depression among Central American immigrants. Psychological Reports. 2000b; 86(3 Pt 2):1237-1240. [PubMed: 10932588]

Hovey JD, Magana C. Acculturative stress, anxiety, and depression among Mexican immigrant farmworkers in the midwest United States. Journal of Immigrant Health. 2000; 2(3):119-131. [PubMed: 16228745]

Iceland J, Scopilliti M. Immigrant residential segregation in U.S. metropolitan areas, 1990-2000. Demography. 2008; 45(1):79-94. [PubMed: 18390292]

Kandel W, Parrado E. Restructuring of the US meat processing industry and new Hispanic migrant destinations. Population and Development Review. 2005; 31(3):447-471. 
Kao G. Social capital and its relevance to minority and immigrant populations. Sociology of Education. 2004; 77(2):172-175.

Kim-Goodwin Y, Bechtel G. Stress among migrant and seasonal farmworkers in rural southeast North Carolina. Journal of Rural Health. 2004; 20(3):271-278. [PubMed: 15298103]

Ko L, Perreira K. "It turned my world upside down": Latino youth's perspective on immigration. Journal of Adolescent Research. 2010; 25(3):465-493. [PubMed: 21909185]

Kochkar, R.; Suro, R.; Tafoya, S. The context and consequences of rapid population growth. Pew Hispanic Center; Washington, DC: 2005. The new Latino south.

Kroenke K, Spitzer RL. The PHQ-9: A new depression and diagnostic severity measure. Psychiatric Annals. 2002; 32:509-521.

Kuperminc, G.; Wilkins, N.; Roche, C.; Alvarez-Jimenez, A. Risk, resilience, and positive development among Latino youth. In: Villaruel, F.; Carlo, G.; Grau, J.; Azmitia, M.; Cabrera, N.; Chahin, T., editors. Handbook of U.S. Latino psychology. Sage; Los Angeles: 2009. p. 213-234.

Lee MA. Neighborhood residential segregation and mental health: a multilevel analysis on Hispanic Americans in Chicago. Social Science \& Medicine. 2009; 68(11):1975-1984. [PubMed: 19359082]

Mair C, Diez Roux AV, Shen M, Shea S, Seeman T, Echeverria S, et al. Cross-sectional and longitudinal associations of neighborhood cohesion and stressors with depressive symptoms in the multiethnic study of atherosclerosis. Annals of Epidemiology. 2009; 19(1):49-57. [PubMed: 19064189]

Marrow H. New immigrant destinations and the American colour line. Ethnic and racial studies. 2009; 32(6):1037-1057.

Massey, D.; Durand, J.; Malone, N. Beyond smoke and mirrors: Mexican immigration in era of economic integration. Russell Sage Foundation; New York: 2002.

Menjivar, C. Fragmented ties. University of California Press; Berkeley and Los Angeles, CA: 2000.

Milette K, Hudson M, Baron M, Thombs BD. Comparison of the PHQ-9 and CES-D depression scales in systemic sclerosis: internal consistency reliability, convergent validity and clinical correlates. Rheumatology. 2010; 49(4):789-796. [PubMed: 20100794]

Moynihan B, Gaboury MT, Onken KJ. Undocumented and unprotected immigrant women and children in harm's way. Journal of Forensic Nursing. 2008; 4(3):123-129. [PubMed: 18798878]

Nicklett EJ, Burgard SA. Downward social mobility and major depressive episodes among Latino and Asian-American immigrants to the United States. American Journal of Epidemiology. 2009; 170(6):793-801. [PubMed: 19671834]

Ornelas IJ, Perreira K, Beeber L, Maxwell L. Challenges and strategies to maintaining emotional health: Qualitative perspectives of Mexican immigrant mothers. Journal of Family Issues. 2009; 30(11):1556-1575.

Ortega A, Rosenheck R, Alegria M, Desai R. Acculturation and the lifetime risk of psychiatric and substance use disorders among Hispanics. Journal of Nervous and Mental Disease. 2000; 188(11): 728-735. [PubMed: 11093374]

Ostir GV, Eschbach K, Markides KS, Goodwin JS. Neighbourhood composition and depressive symptoms among older Mexican Americans. Journal of Epidemiol ogy and Community Health. 2003; 57(12):987-992.

Parrado E, Flippen C, McQuiston C. Migration and relationship power among Mexican women. Demography. 2005; 42(2):347-372. [PubMed: 15986990]

Perreira, K.; Chapman, M.; Potochnick, S.; Smith, TH. Migration and mental health: Latino youth and parents adapting to life in the American south. Final report on the Latino Migration, Health and Adaptation Study. Carolina Population Center; Chapel Hill, NC: 2008.

Perreira K, Chapman M, Stein G. Becoming an American parent: Overcoming challenges and finding strength in a new immigrant Latino community. Journal of Family Issues. 2006; 27(10):13821414.

Perreira K, Potochnick S. Depression and anxiety among first-generation immigrant Latino youth: Key correlates and implications for future research. Journal of Nervous and Mental Disease. 2010; 198(7):470-477. [PubMed: 20611049] 
Radloff L. The CES-D Scale: a self-report depression scale for research in the general population. Applied Pscychology Measures. 1977; 1(3):385-401.

Rasmussen A, Rosenfeld S, Reeves K, Keller A. The subjective experience of trauma and subsequent PTSD in a sample of undocumented immigrants. Journal of Nervous and Mental Disease. 2007; 195:137-143. [PubMed: 17299301]

Rivera FI. Contextualizing the experience of young Latino adults: Acculturation, social support and depression. Journal of Immigrant and Minority Health. 2007; 9(3):237-244. [PubMed: 17265173]

Rivera FI, Guarnaccia PJ, Mulvaney-Day N, Lin JY, Torres M, Alegria M. Family cohesion and its relationship to psychological distress among Latino groups. Hispanic Journal of Behavioral Sciences. 2008; 30(3):357-378. [PubMed: 19444326]

Sentell T, Shumway M, Snowden L. Access to mental health treatment by English language proficiency and race/ethnicity. Journal of General Internal Medicine. 2007; 22(Suppl 2):289-293. [PubMed: 17957413]

Sladkova J. Expectations and motivations of Hondurans migrating to the United States. Journal of Community and Applied Social Psychology. 2007; 17:187-202.

Suarez-Orozco C, Todorova I, Louie J. Making up for lost time: The experience of separation and reunification among immigrant families. Family Process. 2002; 41:625-643. [PubMed: 12613121]

Tienda, M.; Mitchell, F., editors. Multiple origins, uncertain destinies: Hispanics and the American future. National Academy Press; Washington, D.C: 2006.

Torres L. Predicting levels of Latino depression: Acculturation, acculturative stress, and coping. Cultural Diversity and Ethnic Minority Psychology. 2010; 16(2):256-263. [PubMed: 20438164]

Tropp L, Erkut S, Coll C, Alarocon O, Garcia H. Vazquez. Psychological acculturation: Development of a new measure for Puerto Ricans on the US mainland. Educational and Psychological Measurement. 1999; 59(2):351-367. [PubMed: 21415932]

Vega W, Kolody B, Valle R, Weir J. Social networks, social support, and their relationship to depression among Mexican immigrant women. Human Organization. 1991; 50(2):154-162. 
Highlights

- Few studies have assessed how factors at various stages of migration contribute to the development of health problems in immigrant populations.

- We demonstrate how factors prior to, during, and after migration contribute to the development of depressive symptoms.

- High poverty levels prior to migration strongly contribute to the development of depressive symptoms.

- Stress and trauma during migration and discrimination upon settlement further contribute to depressive symptoms.

- Family reunification, social support, and familism reduce the likelihood of depressive symptoms. 


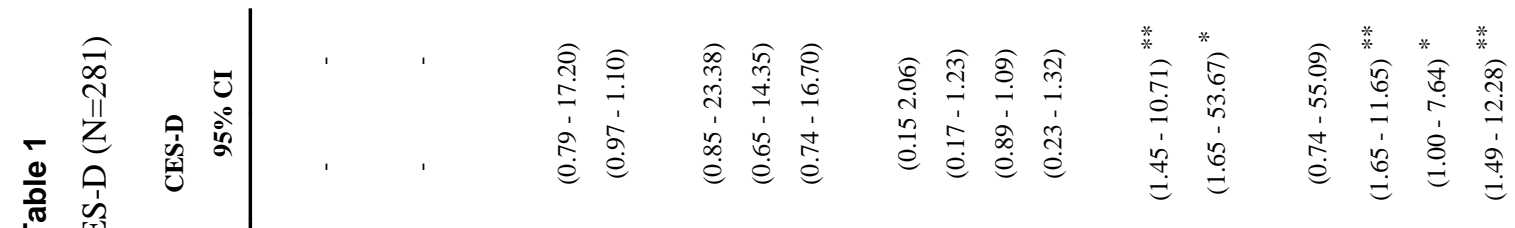

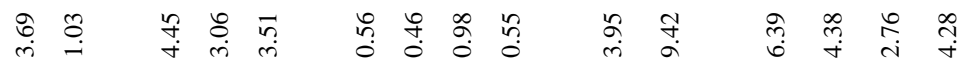

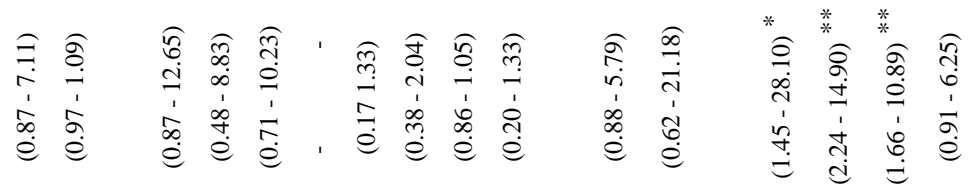

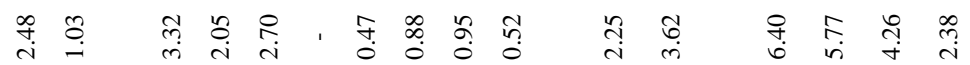

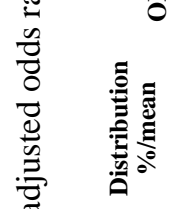




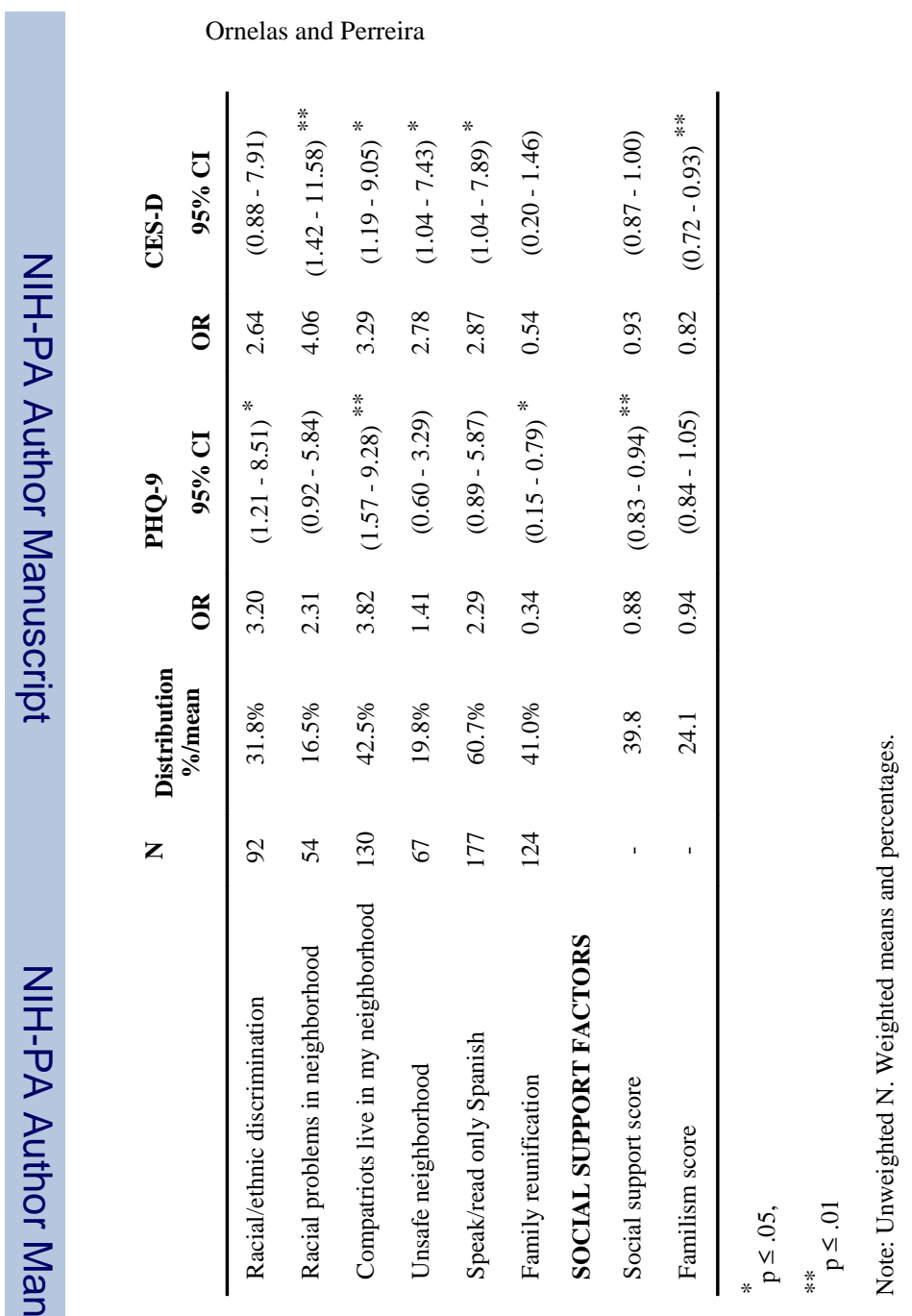

Soc Sci Med. Author manuscript; available in PMC 2012 October 1. 


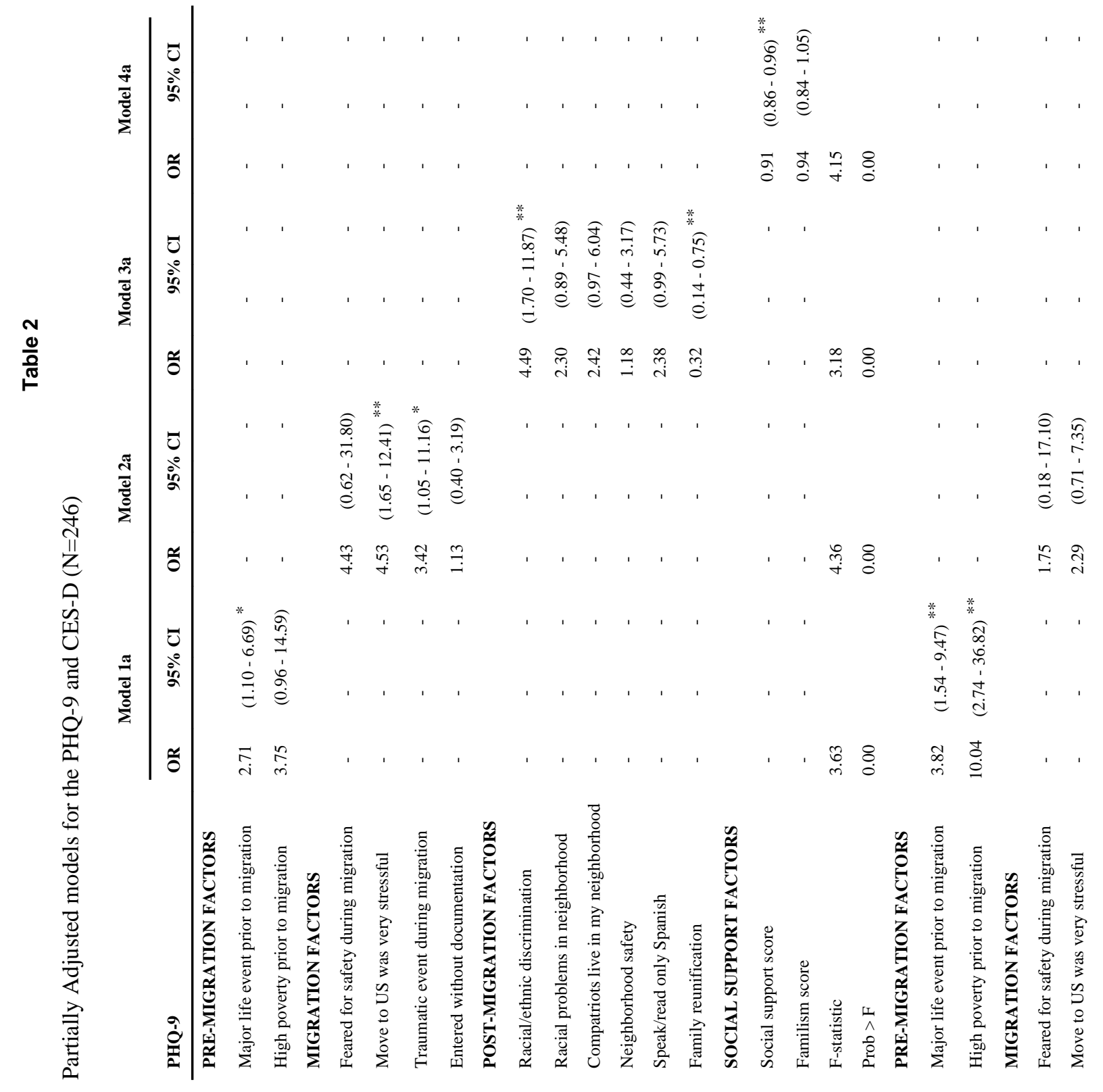




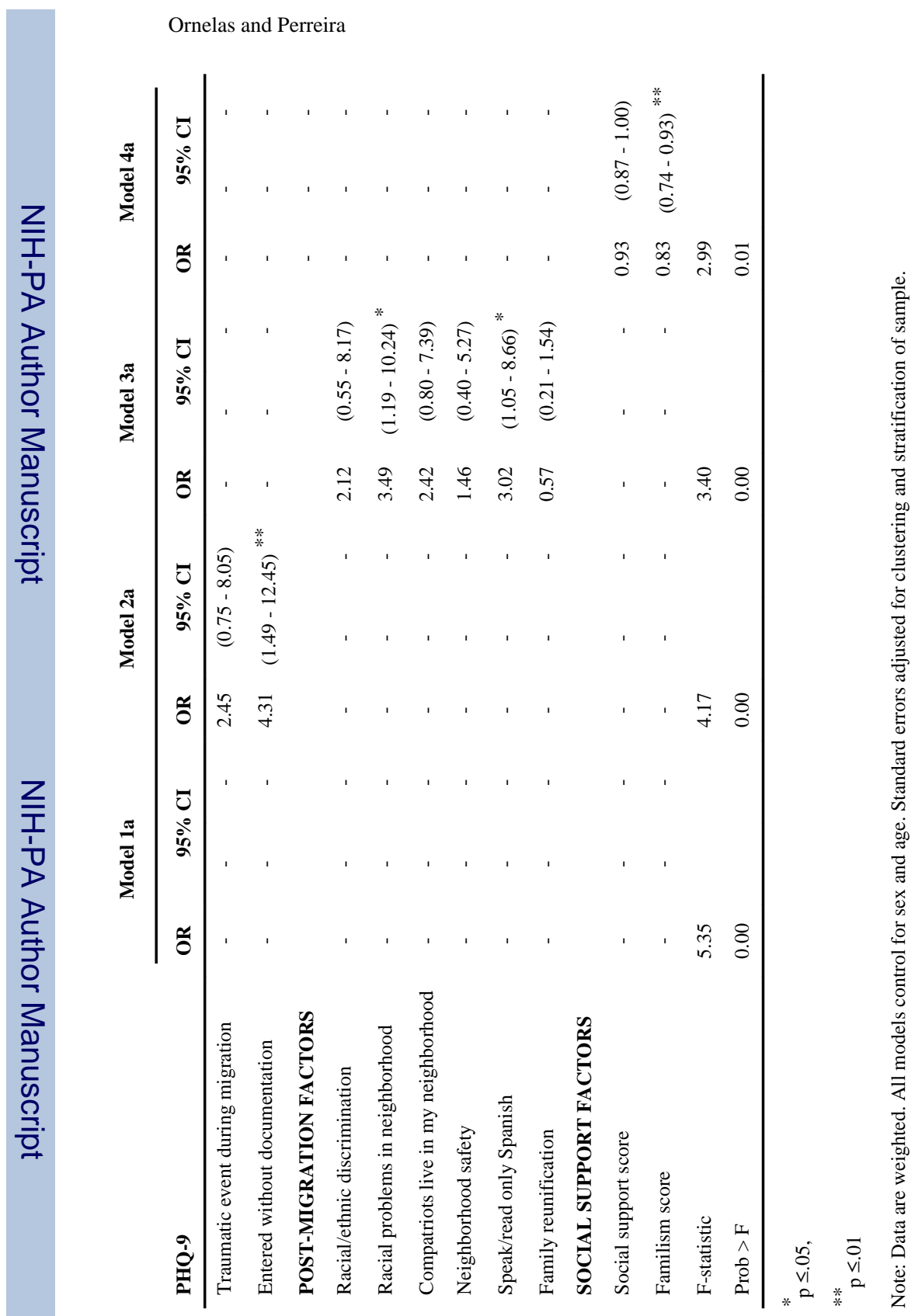

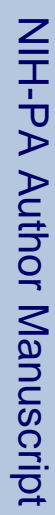

Soc Sci Med. Author manuscript; available in PMC 2012 October 1. 
Table 3

Full models for PHQ-9 and CES-D (N=246)

PHQ-9

\begin{tabular}{|c|c|c|c|c|}
\hline & OR & $95 \% \mathrm{CI}$ & OR & $95 \% \mathrm{CI}$ \\
\hline \multicolumn{5}{|l|}{ PRE-MIGRATION FACTORS } \\
\hline Major life event prior to migration & 1.43 & $(0.563 .65)$ & 2.20 & $(0.726 .71)$ \\
\hline High poverty prior to migration & 2.15 & $(0.548 .57)$ & 5.11 & $(1.0524 .80)^{*}$ \\
\hline \multicolumn{5}{|l|}{ MIGRATION FACTORS } \\
\hline Move to US was very stressful & 5.62 & $(1.96-16.11)^{* *}$ & 2.60 & $(0.77-8.77)$ \\
\hline Traumatic event during migration & 2.29 & $(0.75-7.00) *$ & 1.82 & $(0.55-6.09)$ \\
\hline Entered without documentation & 0.87 & $(0.32-2.39)$ & 2.35 & $(0.75-7.41)$ \\
\hline \multicolumn{5}{|l|}{ POST-MIGRATION FACTORS } \\
\hline Racial/ethnic discrimination & 4.34 & $(1.64-11.47)^{* *}$ & 2.98 & $(0.94-9.47)$ \\
\hline Racial problems in neighborhood & 2.34 & $(0.85-6.48)$ & 5.76 & $(1.55-21.37)^{* *}$ \\
\hline Speak/read only Spanish & 2.51 & $(0.84-7.49)$ & 3.61 & $(0.83-15.69)$ \\
\hline Family reunification & 0.24 & $(0.09-0.63)^{* *}$ & 0.34 & $(0.11-1.05)$ \\
\hline
\end{tabular}

\section{SOCIAL SUPPORT FACTORS}

\begin{tabular}{|c|c|c|c|c|}
\hline Social support score & 0.93 & $(0.88-0.98)^{* *}$ & 0.98 & $(0.91-1.05)$ \\
\hline Familism score & 0.92 & $(0.82-1.04)$ & 0.82 & $(0.72-0.93)^{* *}$ \\
\hline F-statistic & 3.42 & & 3.06 & \\
\hline Prob $>F$ & 0.00 & & 0.00 & \\
\hline
\end{tabular}

Note: Data are weighted. All models control for sex and age.

Standard errors adjusted for clustering and stratification of sample. 